\title{
CRITERIOS DE INTERVENCIÓN Y RECOMENDACIONES DE DISEÑO A SISMO EN LAS ESTRUCTURAS DE PATRIMONIO HISTÓRICO
}

\author{
( CRITERIA FOR INTERVENTION AND RECOMENDATIONS ON SEISMIC DESIGN IN \\ STRUCTURES OF THE HISTORICAL HERITAGE)
}

Antonio J. Mas-Guindal Lafarga, Dr. Arquitecto

Prof. Titular de la ETSAM

ESPAÑA

Fecha de recepción: $21-\mathrm{V}-96$ 409-11

\begin{abstract}
RESUMEN
En este articulo se describen las condiciones particulares de las estructuras históricas sujetas a acciones sismicas.

Las actuales normas sísmicas están hechas desde el edificio moderno de vigas y pilares, siendo los edificios de fábrica sistemas de gravedad que trabajan a compresión, como materiales pétreos sin tracciones.
\end{abstract}

Por otra parte, la aparición de grietas, hasta hoy, era motivo de diagnóstico por ruina.

Nuestro actual conocimiento de los materiales clásicos, sus técnicas y fases de construcción, nos muestran que los edificios antiguos deben ser vistos y analizados desde un punto de vista particular.

Finalmente, se incluyen algunas recomendaciones de diseño sísmico para estructuras de fábrica.

\section{SUMMARY}

This paper describes the peculiar conditions of historic masonry structures subjected to seismic actions.

The present seismic standards govern modern constructures with columns and beams; but masonry buildings are built upon a base of gravity systems, which work on compression, as stone materials with no tractions.

In another hand, the appearance of cracks, until now, was the reason for a diagnosis of decay.

Our present knowledge about classic building materials, technics and construction stages indicates that old buildings must be considered and analysed from a particular viewpoint.

Finally, some recommendations of seismic designs for masonry structures are included.
El mapa geográfico-sísmico, distinto para cada zonay país, ha obligado a reaccionar frente a la agresión sísmica de forma distinta en el espacio y en el tiempo. Los países del centro del continente americano tienen dos aspectos, quizás singulares, sobre las cualidades propias del fenómeno sísmico: por una parte se encuentran en zonas de alto riesgo (Méjico, Guatemala, Colombia) y, por otra, tienen un gran patrimonio histórico. Sismo y patrimonio no se hallan legislados de forma conjunta, y todo lo que se posee son una serie de recomendaciones o apéndices normativos que intentan aportar información y criterios de diseño. En España, este riesgo, se da en las provincias del litoral mediterráneo.

La experiencia que tenemos ante estos hechos provoca, de una parte, una intervención eficaz y, a su vez rápida, en el patrimonio y, de otra, la necesidad de salvaguardarlo desde su misma esencia. Un paseo por las estructuras históricas que han sufrido sismo, se torna un poco desolador' ${ }^{1}$, pues, o bien están en suelo de forma irreversible (fig. 1), o

\footnotetext{
(1) Como muestra se pueden dar los innumerables ejemplos de patrimonio americano sometido, frecuentemente, a sismos importantes, particularmente en los países de Ecuador, Colombia, Guatemala. Méjico. ...etc.
} 
bien han sufrido la agresión, también irreversible, de unos métodos de restauración y unos modos que han desfigurado la cualidad fundamental, que obligaba su respeto, todo ello, por una equivocada concepción de la rigidez estructural aplicable al edificio de fábrica.

Los edificios a los que aquí nos referimos, los pertenecientes al patrimonio histórico, son, en su mayor parte, conjuntos realizados con materiales pétreos, calcáreos o cerámicos que se organizan formando estructuras de gravedad, muros, pilares y bóvedas, en donde su única cualidad es resistir por forma y peso, aspecto éste fundamental para comprender la razón de su estática. Sus elementos carecen de resistencia a flexión, lo que ocasiona, ante la acción dinámica, estados de agrietamiento y desplazamiento de sus masas, con la consecuencia que esto origina en los equilibrios funiculares, generándose situaciones de patología irreversible.

Toda la arquitectura antigua obedece a este modelo de cúpula, empuje y muro de gravedad, pero sabiamente dispuesto. Estructuras muy bien estudiadas, prototipos distintos, difíciles de sistematizar, poco sensibles a la cantidad de carga y mucho a la forma de ser aplicada, ya que la antifunicular de esa carga dará la forma estructural teórica.

Salvo los envigados de madera y artesonados, con la rigidez propia de la barra articulada, que en caso de sismo contribuirán poco a la estabilidad global, toda la estructu-

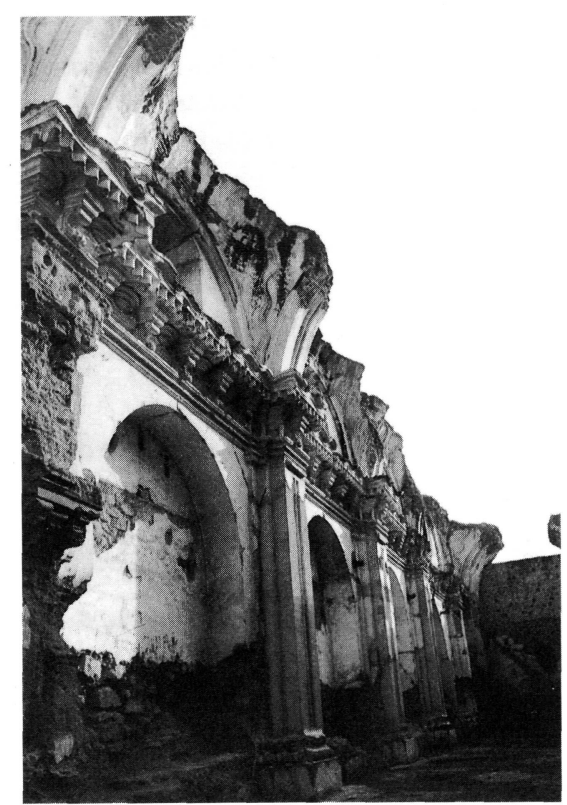

Fig. 1.- Iglesia de El Carmen, de La Antigua (ruatemala. Ruinas debidas a la acción sísmica (1)-3). Foto del autor. ra es un conjunto con rigidez indeterminada, en la que el valor será difícil de concretar, siendo más preciso hablar de recomendaciones constructivas a partir de algunas evaluaciones iniciales.

Hoy se dispone de un moderno sistema de evaluación del riesgo sísmico, donde pueden empezar a predecirse, con alguna precisión, los riesgos de aparición de un sismo, contándose también con gran cantidad de códigos sísmicos especializados para cada país y zona.

Ante este panorama, y dada la dificultad de aplicar normativas, pensadas todas desde la aplicación de fuerzas equivalentes en edificios adintelados de rigidez a flexión (hormigón y acero) y no a edificios históricos de características bien distintas, defendemos, desde aquí, un análisis específico de cada edificio tradicional.

No nos parece que sea el método de análisis la causa de un determinado refuerzo, como puede adivinarse en algunas reparaciones, sino el análisis de un determinado fallo estructural, el que debe generar el método de análisis e intervención. A menudo se observan edificios de valor patrimonial, desfigurados por haber sido sometidos a la implantación severa de esquemas de vigas y pilares de hormigón o acero, sin ninguna apreciación hacia el objeto original. Coexisten en estas soluciones las dudas de una correcta compatibilidad entre la forma nueva trasladada y la antigua original. Esta diferencia inicial en el planteamiento del análisis es fundamental para llegar a unos resultados $\mathrm{u}$ otros.

Efectivamente, la transformación del esquema, de abovedado a adintelado, ofrece la posibilidad de tratarlo bajo norma y modelos establecidos. Ahí, el edificio, ya tiene fuerzas aplicadas en nudos, modos de vibración, tipificándose los nudos de hormigón. No se puede, o no se debe, transformar un esquema estructural de funcionamiento en otro que los códigos y los ordenadores resuelven, porque es muy posible que los resultados que se obtengan tengan poco que ver con la realidad.

El problema de la compatibilidad entre la estructura dañada y su estado precario, muy distinto del original, y la estructura adintelada superpuesta, es una cuestión no resuelta, no debiéndose crear un problema para resolver otro.

En la reparación de una estructura histórica coexisten varios aspectos que no pueden separarse de su propia forma de trabajar. Una estructura adintelada, está perfectamente diferenciada (fig.2), y es fácil operar sobre ella; los pilares, vigas y forjados se diferencian de los cerramientos, coberturas, instalaciones y elementos de acabado. Esta cualidad no existe en la estructura antigua que es, a la vez, cerramiento (muro, contrafuerte), cobertura (bóveda, y más en América, en donde a la forma andaluza no se cubre 
la bóveda) y contiene todos los elementos de acabado². Así, los caminos por los que puede llegar la carga al suelo no están explícitamente determinados (fig. 3). Debe, asimismo, recordarse el efecto negativo que tiene la discontinuidad en muros, pilares, contrafuertes,...etc. de los materiales ( sillería, cerámica, ripios de relleno,...).

Parece razonable actuar desde el objeto y no contra él aplicando así, la frase de Hegel "educar al otro desde el

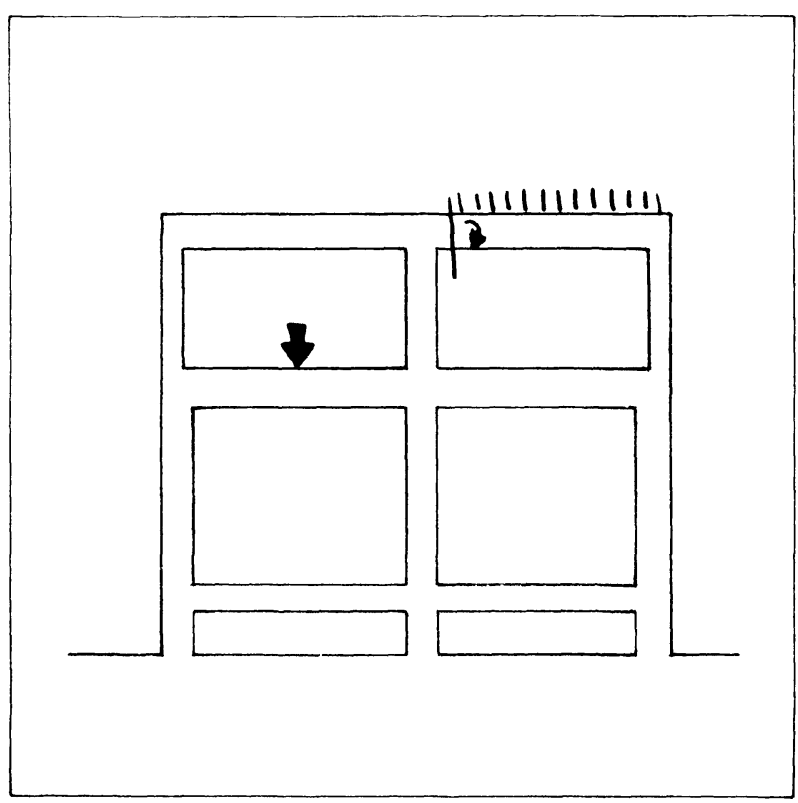

Fig. 2.- Modelo adintelado. Solicitaciones concentradas.

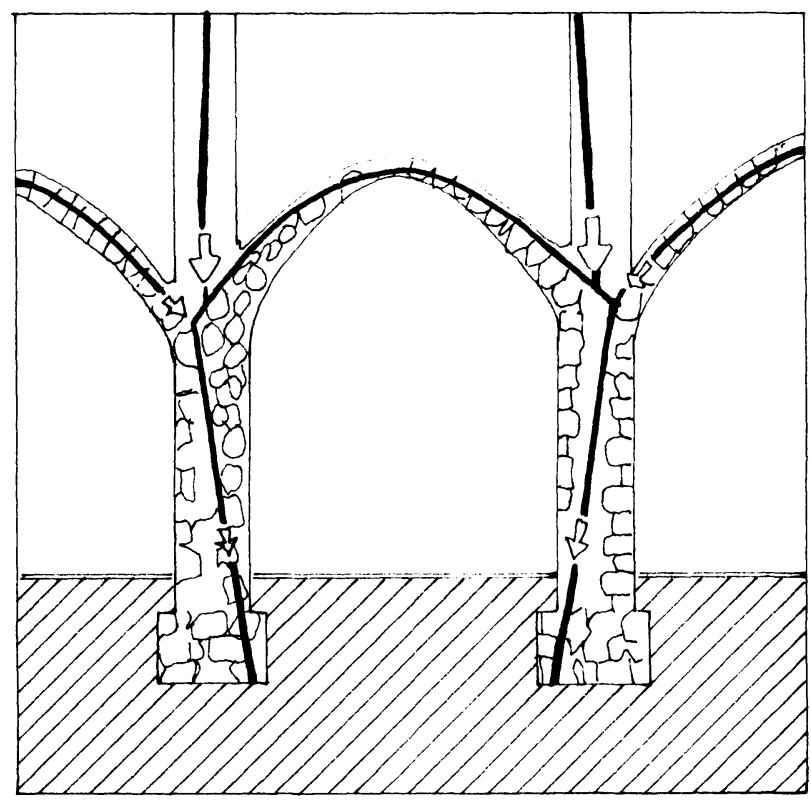

Fig. 3.- Modelo abovedado. Solicitaciones dispersas.

\footnotetext{
(2) El mismo hecho de trabajar a poca tensión, hace dificil definir la travectoria de las solicitaciones.
}

otro", reforzar el edificio sí, pero desde él mismo, desde su forma de trabajar, desde su aspecto, desde su historia, su yacencia.

Estas ideas presiden este artículo, que no pretende desvirtuar el método científico actual a sismo, pero sí llamar la atención sobre el peligro que tal método puede ocasionar si se aplica ,sistemáticamente, a edificios para los que no está pensado.

Cuando una estructura antigua ha sido dañada, caben varias consideraciones, en función de su estado; que esté totalmente arruinada o que lo esté parcialmente.

En el primero de los casos, recrear el edificio con una nueva construcción ex novo que imite la anterior, lleva a un falso histórico, con una base difícil de justificar, y más de convencer, al presunto medio cultural, en el segundo, y siempre que el contexto del edificio esté presente, éste debe repararse a la vez que su memoria. Reconstruir un edificio en su esencia, lleva a considerar, de base, la normativa sísmica específica del sitio, marcando ésta el diseño para proyectar una estructura adintelada sobrepuesta, en la mayor parte de los casos.

Conservar la memoria de un edificio no supone prohibir un cambio de uso. Todos los palacios del renacimiento florentino han cambiado de uso $\mathrm{y}$, sin embargo, conservan su memoria intacta. Por otro lado, un sinfin de edificios históricos han sido sometidos a violentas operaciones de ortopedia que los hacen irreconocibles, aún manteniendo su uso primitivo, todo ello por no haber estudiado el edificio desde él mismo.

A la vista están iglesias que han perdido uno o dos pisos de sus torres en la reparación, o que se les eliminó el pórtico cobijo de entrada para realizar el refuerzo, o éste, en la cimentación, se realizó sin documentación previa sobre la arqueología medieval. La cultura del refuerzo técnico destruyó la cultura material histórica, problema, ëste, complejo, que debe ser contemplado

Todo este panorama nos lleva a reflexionar, junto con el técnico competente, la solución, que debe conducir siempre a no tomar nunca las decisiones de forma unilateral

En la arquitectura tradicional, como en todas, arquitecto e ingeniero dejan su huella siempre que se actúa, y la reflexión sobre lo que debe hacerse no siempre corresponde a ellos solos o, en su caso, sólo a la aplicación de una determinada solución técnica. Desde aquí, creemos en el derecho del edificio a sobrevivir con su aspecto, con la expresión de su propio mecanismo estructural original, hecho que se vulnera con bastante frecuencia.

La primera recomendación que podría establecerse es la de creación de equipos pluridisciplinares que sancionen, o en 
su caso defiendan, las actuaciones. Equipos de arquitectos, historiadores, ingenieros. Ninguno de estos técnicos debe trabajar solo.

\section{Las acciones de partida}

Todo análisis sísmico contiene una serie de pasos basados en los siguientes esquemas:

$\left.1^{\circ}\right)$ Análisis de la respuesta dinámica formulada desde el prototipo creado (estructura real) y el modelo (fig. 4).

$$
\mathrm{F}_{\tau}=\frac{1}{2 \pi} \sqrt{\frac{\mathrm{K}}{\mathrm{M}}} \quad \begin{aligned}
& \mathrm{F}_{\tau}=\text { frecuencia, } \mathrm{K}=\text { rigidez, } \\
& \mathrm{M}=\text { masa }
\end{aligned}
$$

Este prototipo, conlleva concentrar las masas y asignar rigideces estructurales y determinar centros de torsión.

$2^{\circ}$ ) Asimilación del edificio a un oscilador de $\mathrm{n}$ grados de libertad (fig. 5) con $\mathrm{n}$ modos distintos de vibrar para $\mathrm{n}$ frecuencias propias (frecuencias modales). Estas propiedades son invariantes, dependiendo el tipo de respuesta del tipo de suelo.

La excitación tiene múltiples frecuencias, en una estructura rígida, como es el caso de la arquitectura monumental, ésta se corresponde con amplificaciones bajas.

Para una excitación sísmica, debe calcularse la respuesta máxima de estructuras de una frecuencia y un amortiguamiento dados.

$3^{\circ}$ ) Es preciso definir con precisión la trayectoria de las cargas a cimentación.

$\left.4^{\circ}\right)$ La normativa sismo-resistente impone unos parámetros básicos en el análisis en un coeficiente sísmico.

$$
Q_{i}=Z \cdot E \cdot S \cdot R \cdot D \cdot\left(W_{i}\right)
$$

siendo:

$\mathrm{Q}=$ Acción sísmica.

$\mathrm{Z}=$ Factor intensidad de la zona (donde se considera la importancia del edificio.

$\mathrm{E}=$ Amplificación(en términos de frecuencia $\mathrm{y}$ amortiguamiento relativo $\beta$ ).

$\mathrm{S}=$ Suelo

$\mathrm{R}=$ Coeficiente de participación modal.

$\mathrm{D}=$ Factor de ductilidad

$\mathrm{W}=$ Peso en el nivel $\mathrm{i}$

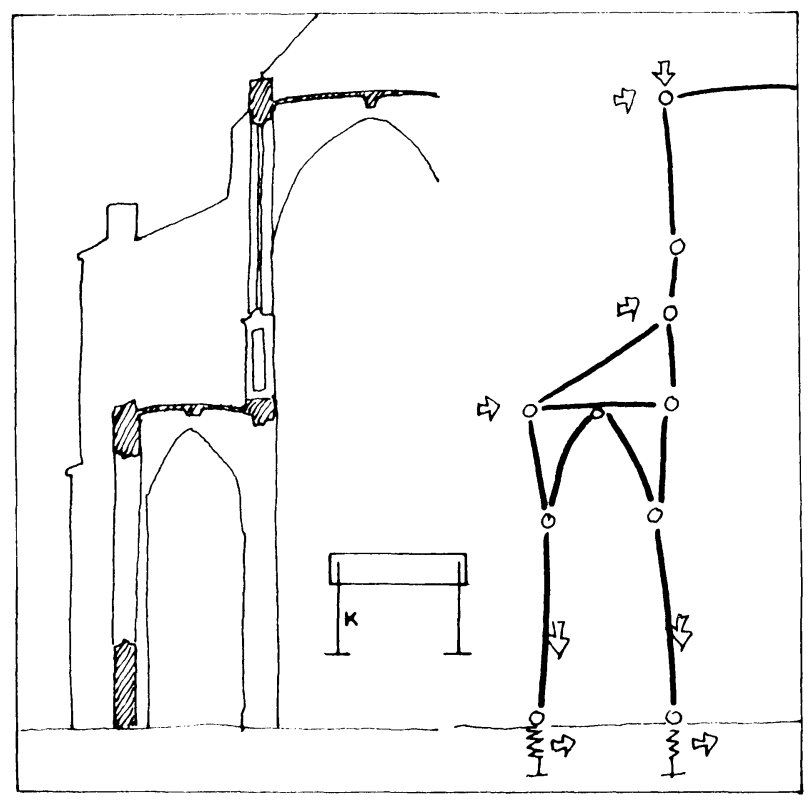

Fig. 4.- Estructura de fábrica real y modelo sismico.

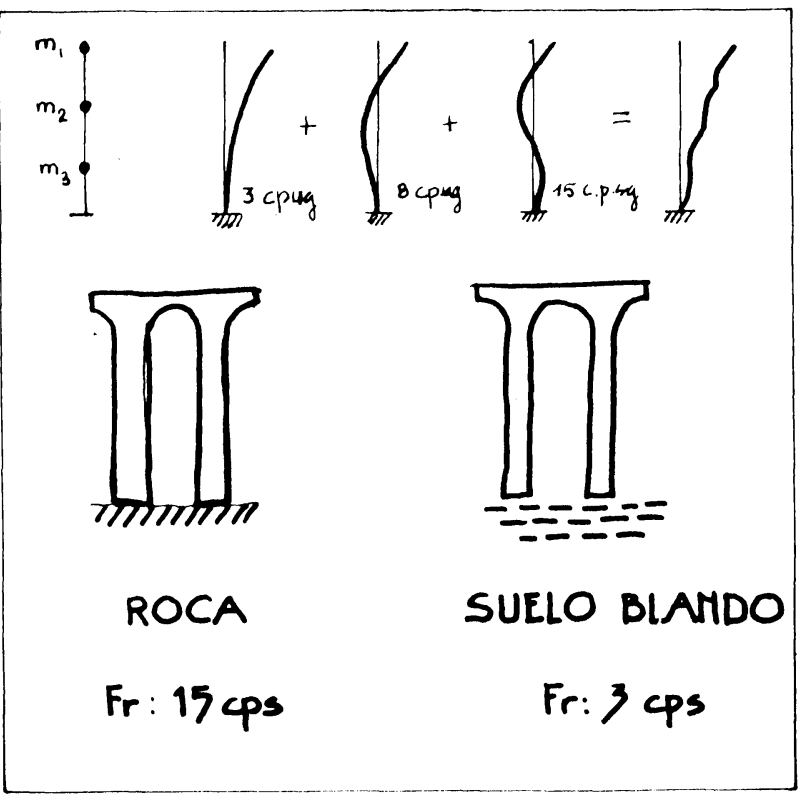

Fig. 5.- Oscilador de tres modelos de vibración. Frecuencia y tipso de suelo.

Con estas acciones se procede al cálculo sísmico, acciones que no siempre se corresponden con la respuesta del edificio de fábrica, pero que sí condicionan el diseño de los elementos de refuerzo. Nada, o muy poco, se sabe de la compatibilidad de estos elementos ex novo de refuerzo en relación a los paramentos de fábrica con los que debe trabajar.

La aparición de un sismo lleva, indefectiblemente, a una redistribución de acciones que deben ser conocidas y determinadas. La combinación de las acciones gravitatorias, viento y sismo, permiten conocer las trayectorias pésimas 
que servirán para diseñar la estructura y/o el refuerzo en su caso.

La realidad es que el comportamiento anisótropo de las estructuras antiguas es causa de severas limitaciones a la hora de modelar el edificio. El nivel de incertidumbre sobre el comportamiento de las fábricas, y su falta de continuidad en los comportamientos, es base suficiente para crear dicha incertidumbre e imposibilita realizar procesos de análisis coherentes.

Las construcciones antiguas contienen materiales de comportamiento muy distinto: piedra, cerámica, madera, ripio de relleno, imperfecciones constructivas y un sinnúmero de obras realizadas durante la vida del edificio, a las que hay que añadir las deformaciones plásticas, durante y después de acabar la obra.

Reparar una bóveda no es sólo calcular sus refuerzos, sino comprender cuáles son sus acciones internas, evaluarlas y conseguir que siga funcionando como en origen.

Los sistemas abovedados en los que hay que intervenir después de acaecido el sismo, suelen ser, en su mayoría, construcciones de hace varios siglos, en donde es dificil conocer la superposición de reparaciones y estados por los que ha pasado la estructura. Es determinante tener una visión lo más exacta posible de como se encuentra el edificio. La determinación de la forma original y la de su geometría trasladada (fig.6), constituye, ya por sí

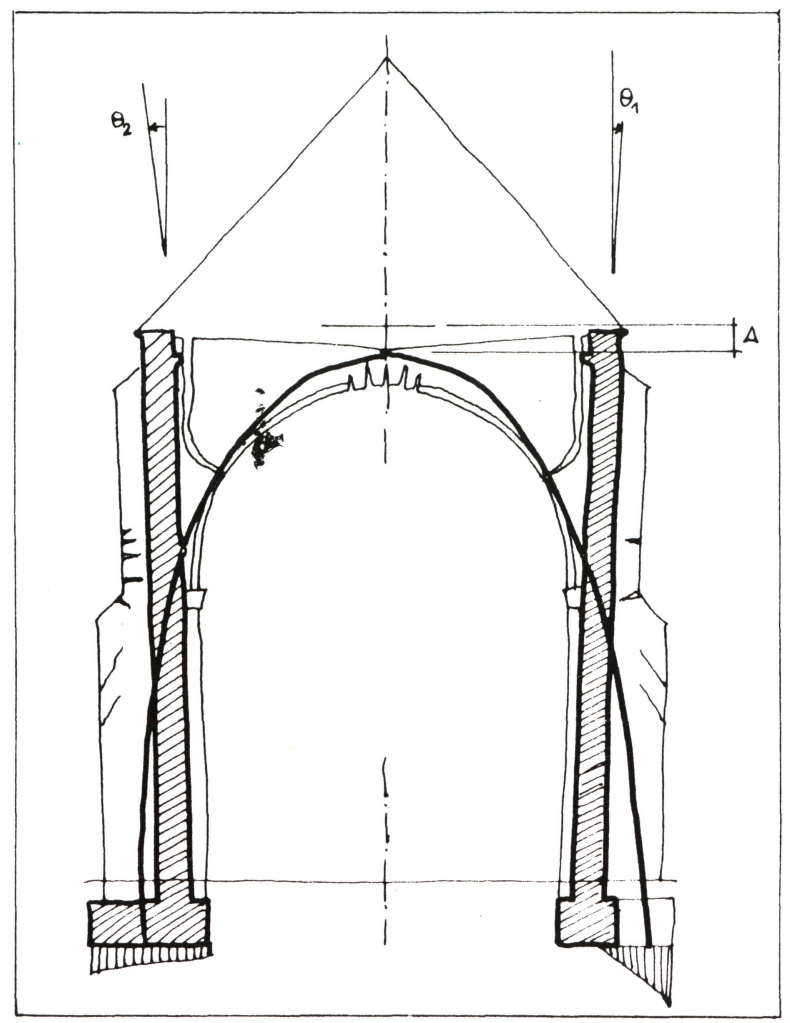

Fig. 6.- Relación entre la geometría trasladada y la posición de las cargas.

(c) Consejo Superior de Investigaciones Científicas Licencia Creative Commons 3.0 España (by-nc) mismo, un problema de especialista. La determinación de los descensos en claves de bóvedas y basas de pilares, los movimientos de los apoyos, los desplomes de pilares y el conocimiento exhaustivo del edificio agrietado son operaciones preliminares necesarias en el punto de partida para el análisis.

Los esquemas fisurativos, aunque $\in$ n sí mismos no predicen como va a comportarse el edificio en el sismo siguiente, sí muestran con elocuencia cómo se ha comportado el edificio ante una agresión conocida y medida. En la mayor parte de los casos, el estado agrietado muestra una manera explícita de trabajar, por la elocuencia de la formación de las articulaciones plásticas. El efecto del sismo se caracteriza por la aparición de grietas a $45^{\circ}$, debidas al esfuerzo cortante que se transmite desde el suelo, y que se evidencian en aquellos puntos en los que la estructura es más sensible, como: claves de arcos, puertas, ventanas, discontinuidades,...etc. Esos puntos son los indicados para crear articulaciones o puntos de reserva plástica, en caso de futuros sismos.(fig. 7).

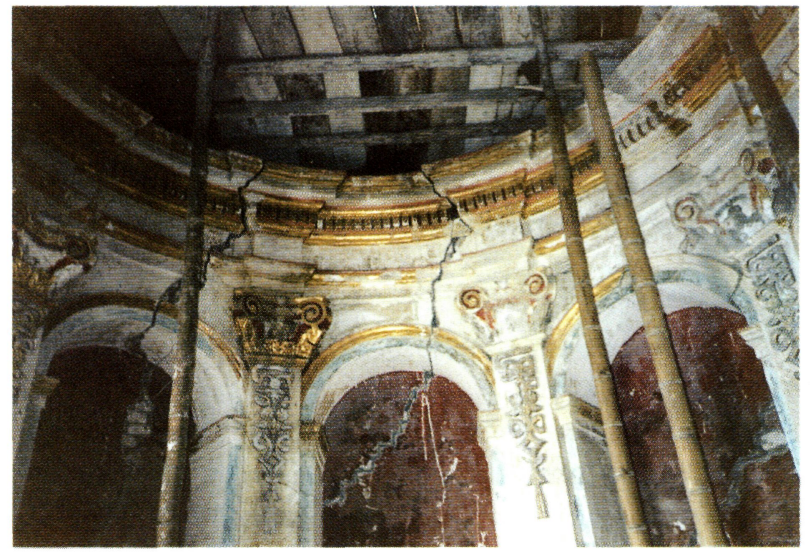

Fig. 7.- Monasterio de San Francisco de Popayán (C'olombia). (irietas a $45^{\circ}$, debidas al efecto sísmico. Foto del autor.

\section{Diseño de vigas}

Las estructuras actuales se proyectan con el criterio de dotar de ductilidad a los nudos en sus acometidas de vigas a pilares. Las recomendaciones apuntan a dotar de una reserva dúctil adicional a los apoyos de vigas. Basándose en la reserva plástica de las zonas de rótulas, éstas experimentan en su entorno grandes deformaciones anelásticas previas a rotura. Hay que tener en cuenta que esta reserva dúctil es difícil, cuando no imposible de conseguir en edificios históricos, ya que los adintelados son siempre de madera y cualquiera que sea su unión al muro, siempre están articulados a él, debido a la imposibilidad de poder estimar uniones rígidas. Esto hace que estas estructuras adinteladas históricas, ante sismo, se comporten como si de muros independientes se tratara, con la única rigidez que le dan los muros transversales, contrafuertes, y los rellenos laterales de bóvedas, que aportan estabilidad 
al conjunto, pero que pueden volverse perjudiciales en la solicitación que nos ocupa, al aportar masa al oscilador.

En hormigón armado, la ductilidad deseada requiere la existencia de desplazamientos fuera del campo de la elasticidad, por lo que la formación de las rótulas exige una longitud determinada que afecta al estribaje del armado en hormigón armado y/o los refuerzos en los nudos de acero. Los distintos códigos aportan diversos criterios de armado en forma de reglas de diseño en aquellas estructuras que pueden ser reguladas bajo norma, hecho que no es el que suele corresponder a un monumento antiguo.

Sin llegar a los criterios específicos de armado contenidos en las normativas (ver bibliografia) la NCS-94 española aconseja, como paso previo para producir estructuras de ductilidad ALTA o MUY ALTA:

-Diseñar vigas preferentemente de canto (no planas).

-Diseñar la armadura de las vigas de forma que éstas plastifiquen antes que el soporte.

-Las secciones de vigas y soportes deben de plastificar antes que el nudo.

-Las secciones deben de fallar antes por elongación del acero que por aplastamiento del hormigón.

\section{Diseño de pilares con rigidez}

La unión viga - pilar es un punto crítico en la respuesta de la estructura y, por ello, ha ocasionado la atención de numerosos trabajos recientes, coordinados entre 1984-89 en EE.UU, Nueva Zelanda, Japón, etc, ... (Ver Bib. final $n^{\circ} 4$ ). Los pilares de piedra, o ladrillo, no tienen resistencia a flexión por lo que son muy sensibles a los efectos de las acciones de cortante horizontal, típicas del sismo.

Igual que en el caso de las vigas, nos permitimos realizar algunas reflexiones, a título de recomendación internacional:

1.- La resistencia de un nudo no debe ser menor que la máxima solicitación que pudiera aparecer en ese nudo, funcionando como mecanismo rótula.

2.- El nudo nunca resistirá menos que el pilar, cuya unión viga-pilar debe ser considerada de forma integral.

3.- Durante la perturbación sísmica los nudos responderán, preferiblemente, en rango anelástico.

Bajo un sismo, las fuerzas pueden ser introducidas en cada nudo viga-pilar con el simple criterio de equilibrio. En el nudo se producen fuerzas cortantes y axiles que deben ser equilibradas. La acción de estas fuerzas provoca efectos en el nudo que se expresan al exterior en forma de fisuras, debido a los mecanismos internos.

Uno de los problemas mayores para el proyectista estriba en la necesidad de que el nudo funcione como se ha pensado, por lo que su definición de proyecto deberá ser la correcta.

\section{Estructuras de pared}

Para proyectar estructuras murales con rigidez a flexión es preciso:

1.- Que el suelo y la cimentación asuman siempre la transmisión de las acciones a la estructura.

2.- Que la fuerza lateral sísmica sea resistida por la pantalla, o el elemento rígido que se prepare al efecto.

3.- Que la disposición de los muros en plantasea simétrica, para reducir al mínimo la posible inestabilidad por torsión, por no coincidir en la línea de acción el centro de masas $(\mathrm{CM})$ y el centro de rotación (CR).

4.- Que exista regularidad en los huecos en vertical y la organización sea a base de cajones continuos.

5.- Tratándose de pantallas ancladas a pórticos, o a muros, a través de forjados deben de estudiarse las interacciones entre ambos.

\section{Edificios de fábrica (Muros y Bóvedas)}

Los edificios históricos no han sido descritos en sus recomendaciones por los códigos de sismo, quizás debido a que el comportamiento de las fábricas puede ser predecido con bastante precisión en el nivel de las cargas de servicio pero con poco en los niveles últimos.

En principio, las hipótesis elásticas de secciones planas no son de aplicación, toda vez que una fábrica, bajo carga excéntrica, pierde su geometría, por lo que requiere un mínimo nivel de ductilidad

Las bóvedas son estructuras de gravedad que funcionan a través de su simple curvatura, o doble (cúpulas), transmitiendo sus empujes a los apoyos que se consideran fijos.

En el caso de la bóveda, su comportamientoes bidireccional, ya que, aunque su sección transversal se supone constante a lo largo de la nave, el rozamiento que existe entre arcos iguales origina traslados de carga y una buena defensa a roturas locales. Aun en equilibrio, una estructurà de fábri- 
ca abovedada habrá presentado un sinnúmero de fisuras, grietas y acomodos, debidas a los descimbrados originales, movimientos del suelo, en sus proximidades a los muros (llamadas por Pol Abraham “Grietas de Sabouret”) (fig. 8).

En estas condiciones, sucede un sismo del que se desconoce su intensidad previamente y colabora, si cabe más, cuantono a la ruina, de la fábrica. Los daños, necesariamente, hay que correlacionarlos con el estado anterior de la fábrica, de tal modo que permita disipar energía a través de rótulas o no.

Una estructura de fábrica, para arruinarse, precisa de la formación de unas rótulas (en arcos) o bisagras (en bóvedas), que permitan la compatibilidad de desplazamientos. Cuatro

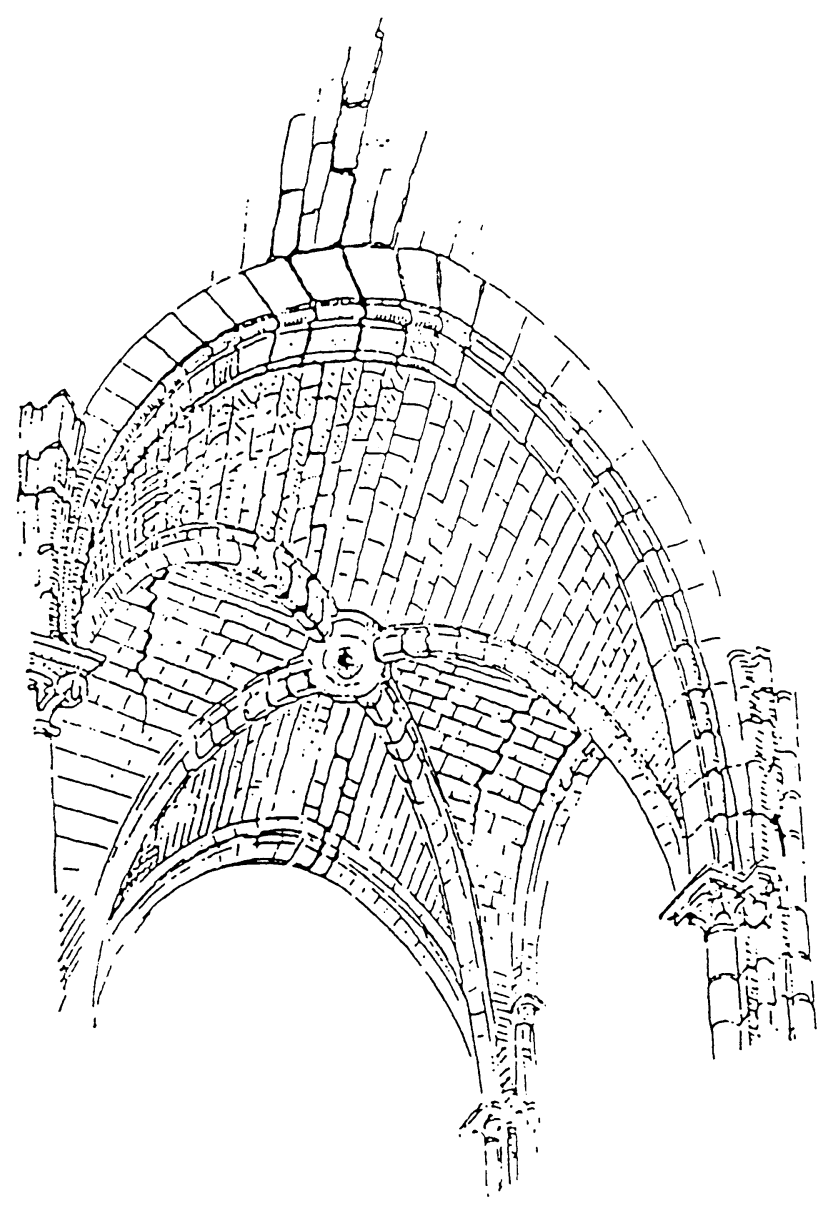

Pol Abraham (1934)

Fig. 8.- Grietas de Sabouret. (Según Pol Abraham, 1934).

\footnotetext{
(3) Las grietas de una bóveda cuatripartita como la de la figura, según Pol Abraham podían ser de tres tipos: $1^{\circ}$ ) Las del cañón de la bóveda en la zona de la clave, debidas a la pérdida de altura al descimbrar; puede asegurarse que todas las bóvedas de cañón las tienen, aunque a simple vista no se aprecien $2^{\circ}$ ) Las paralelas a los arcos formeros o de Sabouret, y $3^{\circ}$ ) Las de separación entre bóveda y muro.
}

articulaciones plásticas son suficientes para que el arco se arruine. En las bóvedas y cúpulas los problemas derivados del acodalamiento entre sillares y rozamiento hacen esta determinación más compleja.

Un arco apuntado encuentra su situación más desfavorable bajo carga al cuarto de la luz. Así se consigue que la línea de empujes salga fuera de la fábrica y el arco se arruine. Una fábrica es sensible a la aplicación de las cargas. Las de sismo, cuando aparecen en forma de esfuerzos cortantes, dejan su huella indicando los puntos de menos inercia por donde las fábricas se han roto.

Estimamos que las fábricas están diseñadas para responder a esfuerzos que ocasionen daños locales; para los sismos no existe este tipo de recomendaciones, ya que las pocas que existen se refieren al proyecto de nuevas construcciones y no a las ejecutadas.

Los habitantes de la Antigua Guatemala, ante el sismo, cambiaron su ubicación por la desesperación de ver sus edificios en el suelo; tal vez aquélla fue una solución inteligente.

Ante edificios que admiten solución recomendamos:

$1^{\circ}$ ) Restitución del sistema de compresiones perdido, debido a las fisuraciones múltiples. Basta realizar inyecciones de mortero de similares características a los existentes. ${ }^{4}$

$2^{\circ}$ ) Estudio de aquellos puntos en los que hay que proporcionar resistencias a tracción inexistentes en fábricas y proceder, por inyección, en dichos puntos. Lo que se busca es introducir con el refuerzo tenaz adicional. Para ello, el análisis a rotura, puede servir de ayuda en la determinación de las cargas últimas de rotura, pero no en la reproducción de los estados críticos de aproximación a aquélla, para lo que se aconsejan modelos más refinados de análisis numérico por elementos finitos. En casi todos los casos, la forma de la rotura explicita, de forma inequívoca, la nueva forma de trabajar la estructura y el análisis del propio daño puede dar la clave de la construcción de las nuevas funiculares del equilibrio.

$3^{\circ}$ ) En elementos abovedados debe de garantizarse la no existencia de desprendimientos locales, hecho que se obvia por la realización de refuerzos. Una rotura local puede desencadenar un efecto en cadena de desequilibrios. Por ello recomendamos armados nominales mínimos que palien ese efecto. Son más importantes las recomendaciones constructivas, desde el punto de vista cualitativo, en lo referente a la forma de respuesta a sismo, que las

\footnotetext{
(4) Estas pastas de aportación notienen porqué ser de resistencia elevada, ya que el trabajo de la fábrica se realiza a baja tensión $\left(2-8 \mathrm{~kg} / \mathrm{cm}^{2}\right.$ de media
} 
cuantitativas, basadas en cuantiosos refuerzos ante una acción que, por su propia naturaleza, es desconocida en el momento de su aparición y en sus efectos (fig.9).

$4^{\circ}$ ) No creemos recomendable reproducir los elementos históricos, bóvedas, a partir de otros materiales que no sean los originales, si bien éstos pueden dotarse de resistencias adicionales.

La construcción de estructuras paralelas debe de evitarse. Un edificio histórico ha de conservar su derecho a existir dentro de los límites de su propia concepción estructural y ésa es la que hay que reforzar.

Las estructuras murales y abovedadas garantizan su estabilidad por la comparación de los elementos debido a su propio peso. Las tensiones de trabajo son bajas, del orden de 1/10 de la resistencia de rotura, y la mayor parte de la estructura trabaja a 1/100 de esa resistencia, hecho que las hace suficientemente seguras. Lo realmente importante es que la estructura mantenga la forma correcta, por lo que el problema es, no tanto de resistencia como de geometría. Debe de asegurarse que los desplazamientos no van a progresar, ya que volver a la situación anterior a fisuración, en estructuras de fábrica, es del todo imposible.

Un edificio antiguo es más rígido si se compara con uno adintelado de dimensiones equivalentes, por lo que responde con un régimen de frecuencias altas o períodos bajos, lo que hace que acompañe al suelo sobre el que se apoya en la excitación (fig. 10). Cuandoéste no es lo suficientemente rígido, caso de suelos arenosos, o anegados, etc... la

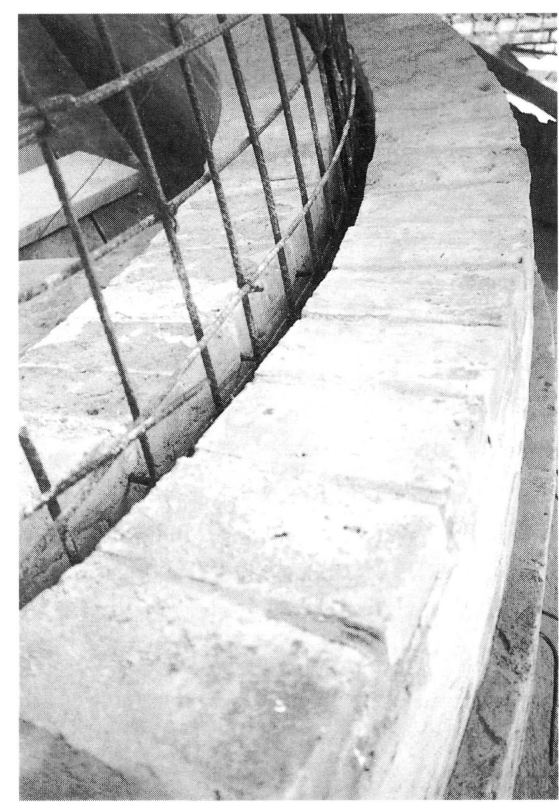

Fig. 9.- Reconstrucción de las bóvedas del Monasterio de San Francisco de Popayán (Colombia). Dr. Arquitecto A. Mas-Guindal. (Foto del autor).

frecuencia disminuye y el edificio puede volverse más vulnerable. De ahí que no sea correcto un análisis del edificio sin el del suelo sobre el que se apoya.

Si una estructura está agrietada, pero no detecta estados de deformación en curso (que puedan ser evidentes por instrumentación adecuada, tales como fisurometría,

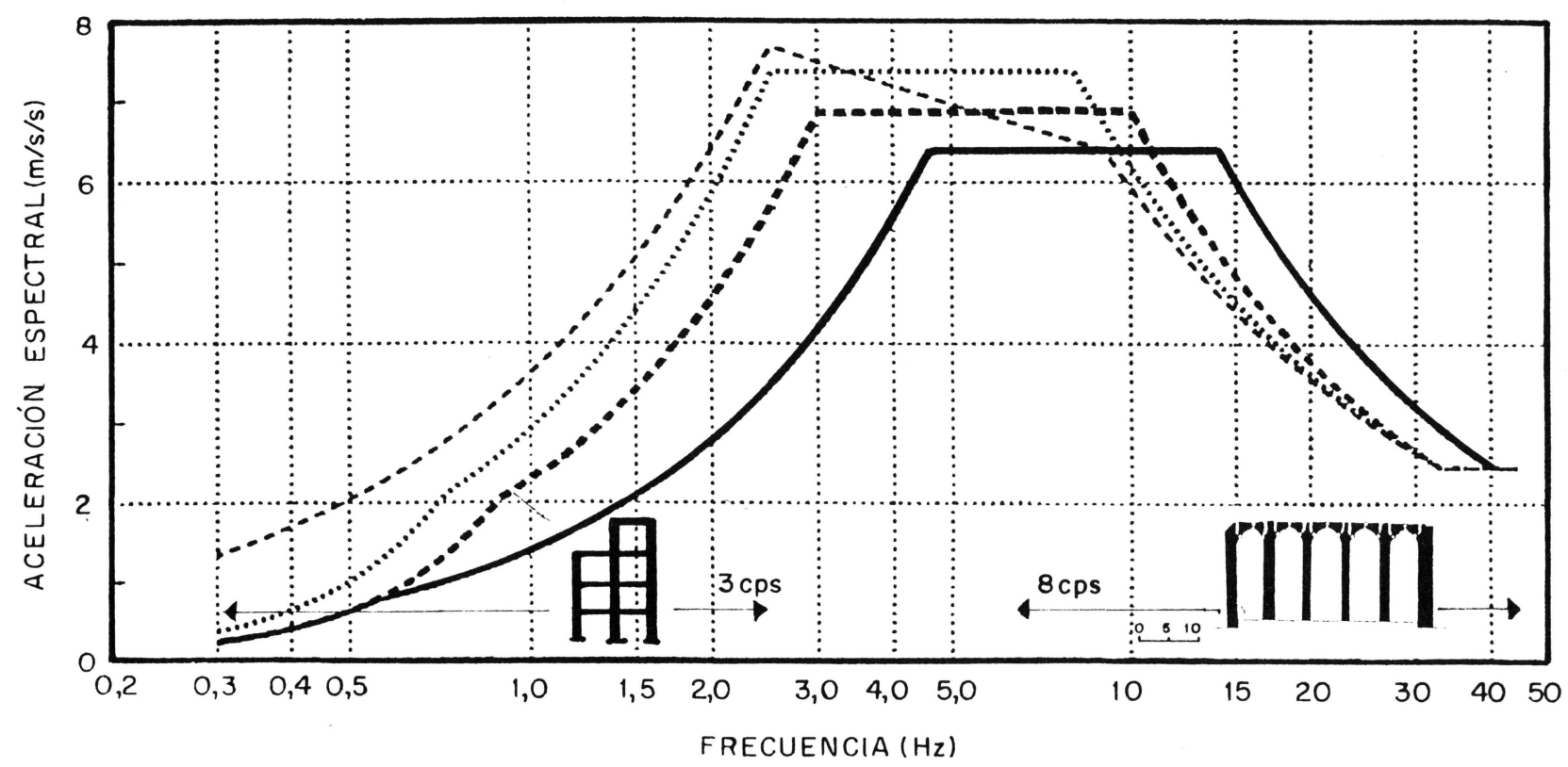

DURO MEDIO FLOJO USNRC (Guía Reg. 1.60

Fig. 10.- Gráfico de frecuencia-aceleración espectral para distintos tipos de suelo. Posición de los edificios adintelados y abovedados. 
extensometría, etc., ...) ésta es segura; simplemente trabaja de forma distinta a como fue pensada, pero eso no justifica ni su ruina ni costosas reparaciones que afectan, en su resultado, a la forma de trabajar y a su imagen.

Las grietas que aparecen por el sismo deben servir de base para la realización de estudios de estabilidad que justifiquen y verifiquen, en su caso, la nueva realidad estructural. De ellos dependerá la continuación del refuerzo oel no actuar.

Los asientos irregulares pueden conducir a cambios notables en la geometría, sobre todo en los elementos altos (torres). $\mathrm{Si}$ las torres de fábrica han sufrido desplomes por otras causas, se encuentran en condiciones especialmente propensas para sufrir los efectos sísmicos de forma fatal.

La construcción mural es, en su mayor parte, de adobe en áreas rurales. La experiencia demuestra que una mejora en la calidad del mismo no redunda en un beneficio de comportamiento a sismo, por lo que no es una buena decisión proceder a sustituciones masivas. El adobe tradicional tomado con morteros de cal grasa, proporciona buenas resistencias a compresión.

Dado que los fallos se deben a defectos de configuración y esquema estructural, resistencia baja del terreno de cimentación, muros no conexos, mala ejecución, etc,... podemos ofrecer las recomendaciones siguientes:

$\left.1^{\circ}\right)$ El edificio debe estar perfectamente arriostrado, por lo que los muros transversales deben estar próximos.

$2^{\circ}$ ) Las fábricas de los edificios dañados deben de revisarse, pudiendo, en su caso, aumentarse de dimensiones en sus secciones.

$3^{\circ}$ ) Los muros deben atarse con un cadena de hormigón armado que no se deforme, para contribuir a la solidaridad de los muros.

$\left.4^{\circ}\right)$ Los cimientos de los edificios dañados deben de revisarse, pudiendo, en su caso, aumentarse.

$\left.5^{\circ}\right)$ Debe usarse la técnica de la sustitución y relleno antes que la demolición total del elemento.

$\left.6^{\circ}\right)$ Los dinteles se reforzarán, preferentemente, por ambas caras.

Algunas normas recomiendan que las cadenas que atan los muros se calculen para los esfuerzos normales máximos producidos por el sismo. Se evitará, en todos los nudos, el posible pandeo de las barras.

Es recomendable utilizar un mismo material en cada construcción y que los coeficientes de elasticidad de los diferentes materiales sean lo más parecidos posibles.
En las mamposterías se evitarán materiales en los que el mortero de cemento se adhiera mal. El ladrillo sílicocalcáreo no es aceptable.

Los muros de gran longitud han de estar arriostrados con muros transversales y los huecos de puertas y ventanas deben realizarse al tiempo que la mampostería, para evitar zonas de discontinuidad

La continuidad en los forjados aporta buenos comportamientos y contribuye a arriostrar el edificio. La edificación tradicional se basa en vigas apoyadas de madera que, ante el sismo, se desorganizan, por lo que la obra de consolidación ha de cuidar este efecto.

\section{BIBLIOGRAFÍA}

(1) UNIFORM BUILDING CODE, 1985: Edition International Conference of Buildings Officials. - Whittier, CA, 1985

(2) NATIONAL EARTHQUEAKE HAZARDS REDUCTION PROGRAMA (NEHRP), RECOMMENDED PROVISIONS FOR THE DEVELOPMENT OF SEISMIC REGULATION FOR NEW BUILDINGS, 1985 EDITION: Building Seismic Safety Council.- Washington, 1985.

(3) DESIGN CRITERIA AND GROUND MOTION EFFECTS ON THE SEISMIC RESPONSE OF MULTISTORY BUILDINGS: J.C. Anderson and F. Naeim: ATC 10-1, Critical Aspects of Earthqueake Ground Motion and Building Damage Potential, Applied Technology Council.- Palo Alto, CA, 1984

(4) NCS-94."NORMA DE CONSTRUCCIÓN SISMORRESISTENTE".

(5) REINFORCED CONCRETE BEAM-COLUMN JOINTS UNDER SEISMIC ACTIONS: T. Paulay, R. Park, and M.J.N. Priestley.- JACI.- Nov., 1978.

(6) THE DESIGN OF DUCTILE REINFORCED CONCRETE STRUCTURAL WALLS FOR EARTHQUAKE RESISTANCE: T. Paulay: EERI Earthquake Spectra 2, $n^{\circ} 4$.Oct. 1986.

(7) DIAGONALLY REINFORCED COUPLING BEAMS OF THEAR WALLS: T. Pauly and J.R. Binney.- Paper $n^{\circ}$ 26.Shear in Reinforced Concrete, ACI Publication SP-42, Vol. 2.- 1974.

(8) ANSYS-ENGINEERING ANALYSIS SYSTEMS: Swanson Analysis Systems, Inc.- Houston, PA.

(9) DYNAMIC EFFECTS OF EARTHQUAKES: R.W. Clough.Trans. ASCE 126, Part. II.

(10) MINIMUN DESIGN LOADS FOR BUILDINGS AND OTHER STRUCTURE (ANSI A58.1-1982): American National Standard Institute, Inc.- New York. The latest edition in the 1982 Edition. 
14

Informes de la Construcción, Vol. 48 n 443, mayo/junio 1996

(11) DETAILS AND DETAILING OF CONCRETE REINFORCEMENT (ACI 315-80): ACI Committee 315.JACI 83, nº 3.- May-June 1986.

(12) REINFORCED MASONRY DESIGN: R.R. Scheneider and W.L. Dickey.-2nd Edition, Prentice-Hall.- Englewood Cliffs, NJ, 1987.

(13) THE STONE SKELETON: Jacques Heyman: Cambridge University Press, 1995.

(14) METHODS FOR THE ANALYSIS AND SAFETY EVALUATION OF MASONRY STRUCTURES SUBJECTED TO SEISMIC ACTIONS: (Spatial structures heritage, Present and Future, 1995. Actas. SGE Ed. Gian Carlo Giuliani,Pavova): G.Croci, P. D’Astia, A. Viskovic.

(15) DINAMIC CRITERIA APPLICATIONS FOR ANALYSIS OF THE STATIC AND DYNAMIC SUFFICIENCY OF MASONRY STRUCTURES: (Spatial structures heritage, Present and Future, 1995. Actas. SGE Ed. Gian Carlo Giuliani,Pavova): b. Leftheris, M.E. Stavroulaki, E.T. Zanaki.

(16) LIMIT ANALYSIS OF MASONRY DOMES. A NUMERICAL PROCEDURE: (Spatial structures heritage, Present and Future, 1995* Actas. SGE Ed. Gian Carlo Giuliani,Pavova): S. BriccoliBati, M. Paradiso, G. Tempesta.

\section{Publicaciones del IETCC/CSIC}

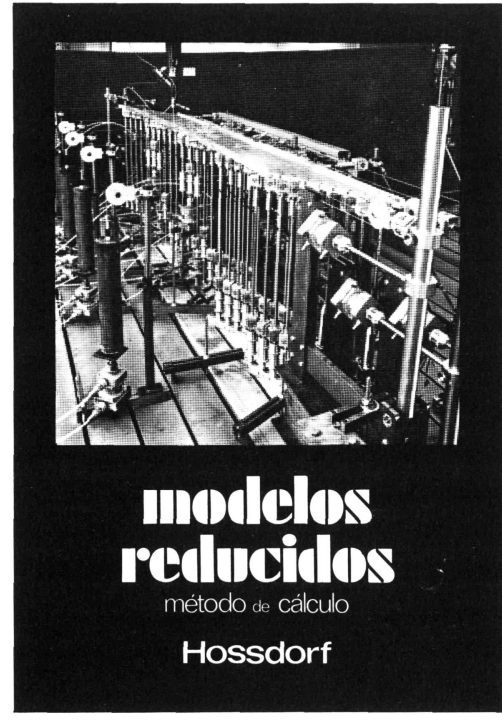

Modelos reducidos. Método de cálculo

H. Hossdorf, Ingeniero Civil

La técnica de los ensayos en modelos reducidos de estructuras sufre hoy dia una decisiva metamorfosis. Hasta hace poco era un medio más bien de artesa nia, que no siempre era tomado en serio por los académicos teorizantes para comprender el comportamiento resistente de las estructuras complejas y al queno resistí de las estructuras complejas y a que se acudio las mas de las veces, como a un ultimo remedio debido a sus indisculibles insuficiencias. Sin embargo, en poco indiscutibles insuficienconexión con los ordenadores digitales, se ha transcormado en un instrumento cienticamento valoso, Un volumen encuadernado en cartoné plastificado
con lomo de tela de $17 \times 24 \mathrm{~cm}$, compuesto de 250 páginas, 158 figuras y fotografias.

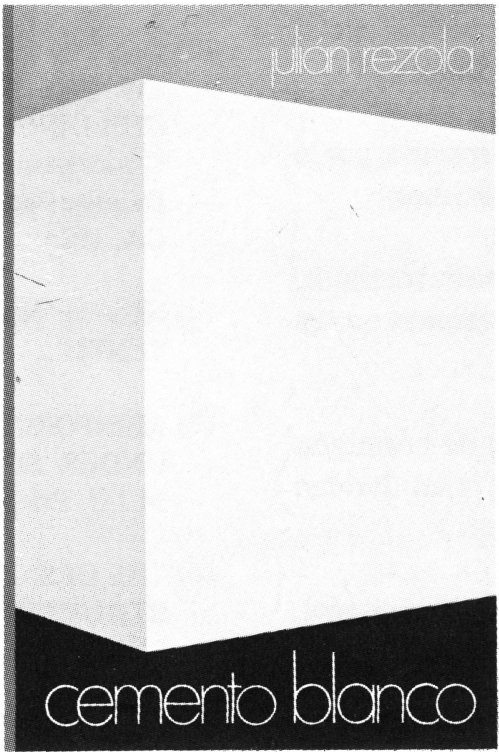

Cemento blanco

Julián Rezola

Ingeniero Químico Dipl. I. Q. S.

Sabido es que existe una extensa y documentada bibliografia sobre el cemento gris: en cambio, no puede decirse lo mismo acerca del cemento portland blanco, ya que los escritos existentes se refieren tan sólo a algunas peculiaridades que le distinguen de aqué

El autor nos ofrece sus profundos conocimientos y su larga experiencia tanto en laboratorio como en fabricación.

La parte descriptiva del libro se complementa con gráficos, diagramas y fotografías de gran utilidad, destinados a conseguir la aplicación apropiada de este aglomerante.

Un volumen encuadernado en cartoné policerado, de $17,4 \times 24,3 \mathrm{~cm}$, compuesto de 395 páginas, numerosas figuras, tablas y ábacos.

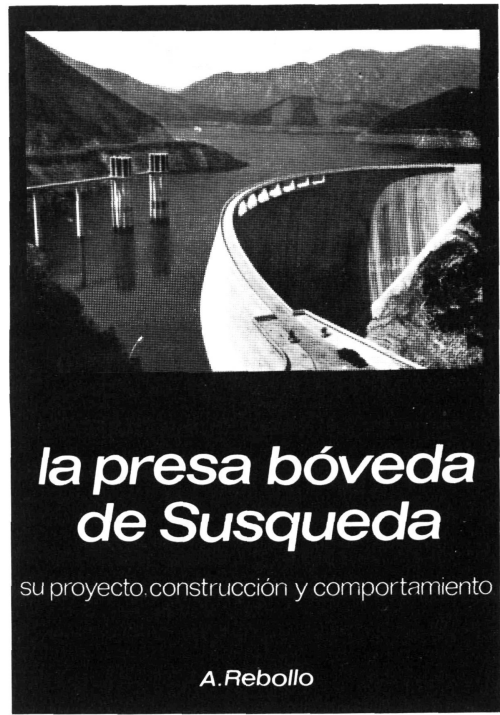

La presa bóveda de Susqueda

A. Rebollo,

Dr. Ingeniero de Caminos

El esfuerzo del constructor de presas se sitúa por su pretension de perennidad, a contracorriente de las tendencias de la civilización actual, caracterizada por lo fungible. Pueden evocarse las 10.000 grandes. presas en funcionamiento o en construcción que estan envejeciendo y reclaman los cuidado gerontologicos para mantener y perfeccionar su servicio y garantizar su inalienable pretension de perennidad. En la medida en que todas nuevas obras, grandes o pequeñas, son portadoras de riesgos ecologicos y, a veces, catastroficos, que aumentan con el envejecimiento, la gerontologia de las presas es todo un emplazo. La accion adelantada de Arturo Rebollo en este terreno marca un camino a seguir para todos los que aman su propia obra con la devoción paternal que èl ha puesto en Susqueda.

Un volumen encuadernado en cartoné plastificado con lomo de tela, de $18 \times 24,5 \mathrm{~cm}$, compuesto de 408 páginas, 330 figuras y fotografias y 39 tablas. 